\title{
Legal storm brewing over Texas forced life-support case
}

$\mathrm{T}$

he husband of an allegedly brain-dead, pregnant Texas woman is suing the hospital that is keeping her on life support, as ethical debate around the case intensifies.

Erick Munoz is seeking a court order to stop all life support for his wife Marlise, who was 14-weeks pregnant when her husband says doctors at John Peter Smith Hospital in Fort Worth declared her brain-dead.

More than a month later, Marlise remains tethered to machines in the hospital's intensive care unit in a bid to sustain her still-living fetus - a fate her family says runs contrary to both her previously expressed wishes and their own.

"Marlise Munoz is dead, and she gave clear instructions to her husband and family - Marlise was not to remain on any type of artificial 'life sustaining treatment,' ventilators or the like," states the lawsuit filed Jan. 14.

Hospital officials claim it's illegal under Texas law to withdraw such treatment from a pregnant patient, regardless of any advance directive.

"Our position remains the same; we follow the law," says Jill Labbe, vice president of communications and community affairs at the hospital. "The courts are the appropriate venue to provide clarity, direction and resolution in this matter."

But a lawyer who helped draft the law in question disputes Labbe's claim. "The hospital has the law wrong," in both intent and letter, says Tom Mayo, an associate professor of law and expert in medical ethics at Southern Methodist University in Dallas. "What needs to be underscored is that the hospital did have a choice and Texas law did not compel this."

Under the Texas Advance Directives Act, hospitals that withdraw life support from a pregnant patient stand to lose legal immunities in their handling of the case - in short, the practice is discouraged, but not illegal.

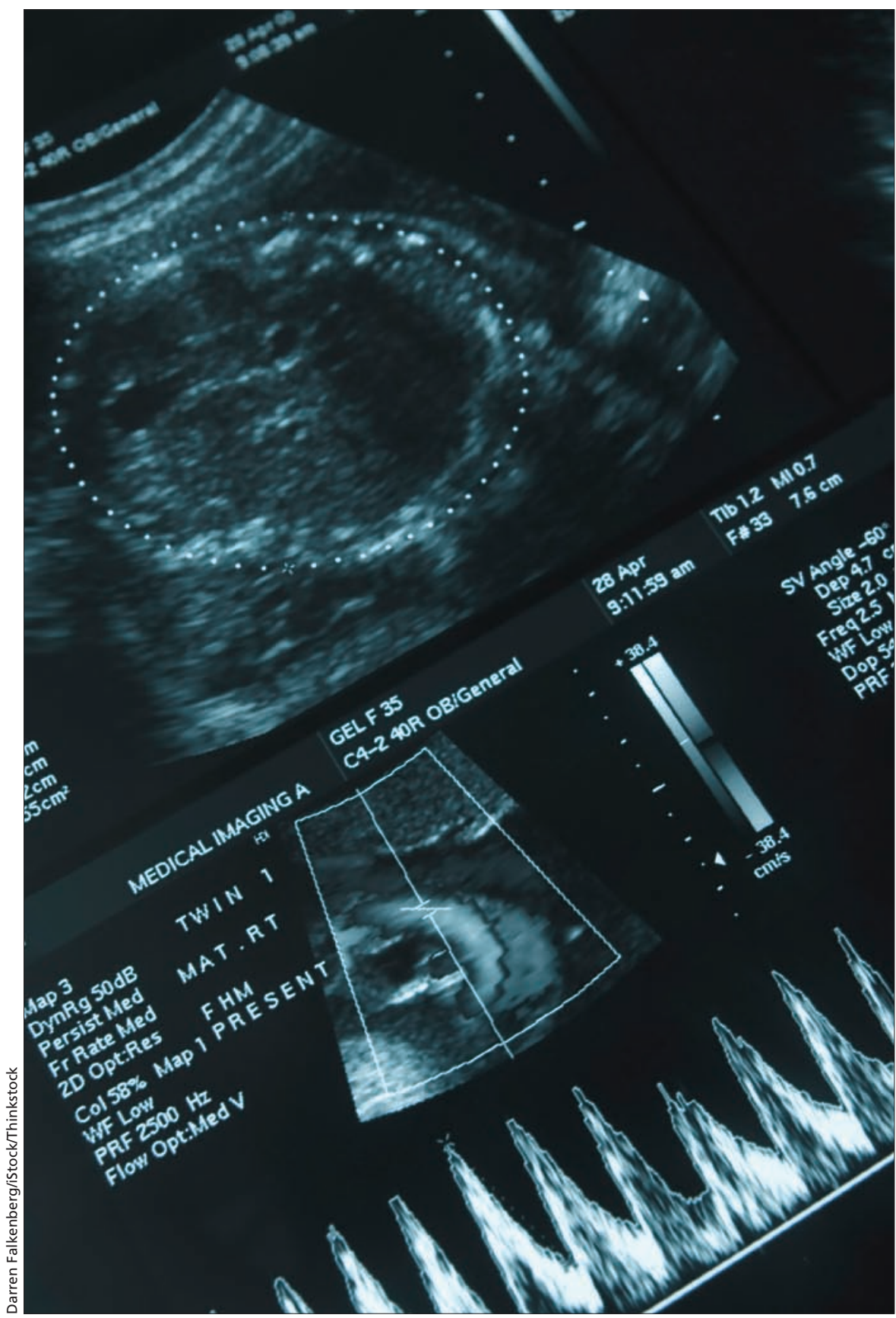

A Texas hospital is keeping an allegedly brain-dead, pregnant woman on life support to sustain her fetus against the wishes of her family.

"If you have a patient who is braindead and a family that wants life support stopped, I should think the legal risks [of withdrawing that support] are pretty low and the need for immunities would be equally low," explains Mayo.
Moreover, the act was written to govern treatment of living patients with terminal or irreversible conditions, he says. "If Marlise is brain-dead, she's dead. We don't talk about corpses having a condition and we don't treat them, 
which is an additional reason why this pregnancy exclusion can and should be disregarded."

In his suit, Erick Munoz likewise challenges that his wife cannot possibly be a "pregnant patient" because she is legally dead and as such her body should be released to her family for burial.

Mayo says there are similar statutes in some 30 states, none of which directly address the "grotesque possibility" of using a dead woman's body to incubate her fetus over the objection of family.

As such, some ethicists question whether Marlise is truly brain-dead, particularly as the hospital hasn't released her medical records to the family.

"I' $m$ wondering if we're going to hear in days ahead that she's not braindead or the hospital doesn't think she is," says Kerry Bowman, bioethicist at the University of Toronto in Ontario. "I feel like there might be a missing piece here."

Even so, it's shocking that "the tem- plate that we always use to make health care decisions - autonomy or substituted autonomy — was thrown right out the window in this case."

Other ethicists, and a leading prolife lobby, argue it's reasonable to weigh the autonomy of a dead woman against the interests of a living fetus.

"You've got the potential of major benefit to the unborn child if it survives and very little downside to keeping [the mother] on life support until you could deliver the baby at a reasonable time," says Margaret Somerville, founding director of McGill University's Centre for Medicine, Ethics and Law in Montréal, Quebec.

Somerville includes cases where the fetus may face serious disability due to its difficult gestation. Otherwise, "what you're saying is that being dead is better than having a life that involves disability or suffering."

But according to Arthur Schafer, director of the Centre for Professional and Applied Ethics at the University of Manitoba in Winnipeg, such balancing tests often overlook the possibility of posthumous harm.

Although Marlise "can't be harmed in the sense of experiencing pain ... treating her body in a way she would have found undignified or leaving a distressing memory of her with her family" constitutes a kind of harm.

"There certainly will be an emotional burden and, in the States, maybe a huge financial burden on the family," Schafer adds.

Labbe says the hospital's financial department "will pursue its customary process for identifying payers and reimbursement."

Erick Munoz has asked the court for an expedited decision in the case. Lauren Vogel, CMAJ

CMAJ 2014. DOI:10.1503/cmaj.109-4704 